\title{
The Comparison of Monte Carlo Algorithms Applied for Off-Lattice Models of Polymer Chains
}

\author{
A. Kuriata, D. Gront, A. Sikorski* \\ Department of Chemistry, University of Warsaw \\ Pasteura 1, 02-093 Warszawa, Poland \\ *E-mail: sikorski@chem.uw.edu.pl
}

Received: 16 November 2016; revised: 23 November 2016; accepted: 24 November 2016; published online: 19 December 2016

\begin{abstract}
We designed two simplified models of macromolecular systems. Model chains were built of united atoms (statistical segments): the first one was a bead-spring model while in the second one beads were connected by bonds of constant length. The only potential introduced was the excluded volume and thus the system was athermal. Monte Carlo simulations of these models were carried out using Metropolis-like algorithms appropriate for each model: the one-bead displacement and the backrub algorithm. The scaling analysis of the chain's static and dynamic properties was carried out. The universal behavior of the chain's properties under consideration was found and discussed. The efficiency of both algorithms was compared and discussed.
\end{abstract}

Key words: Backrub algorithm, computer simulation, Monte Carlo method, off-lattice models, polymer dynamics

\section{INTRODUCTION}

Recent decades have witnessed rapid development of simulation methods applied to the study of polymer and biopolymer systems properties [1]. The complexity of such systems makes theoretical considerations very difficult and computer simulations were found useful for these studies. Some interesting results were obtained up to date; however, for most biopolymer systems, models were rather complicated and contained an enormous number of parameters and, therefore, it was sometimes difficult to judge which of them are really important for obtaining proper results. This was the main reason for designing and studying coarse-grained models of chain molecules in biological systems.

The model studied in this work was designed for studies of mucus. Mucus is a very important element of the innate defense system as it immobilizes and excretes external hostile substances and organisms; beside protection it can serve as a lubricant [2-4]. Soluble mucus is composed mostly of water (more than 90\%) and its key gel-forming agents mucins. Mucins are large extracellular, highly glycosylated proteins that play a role in the human immune system by forming a selective molecular barrier - the aforementioned mucus and are a target of cancer treatment research. Theoretical studies of mucus properties are therefore necessary to enhance the ongoing effort for mucin-based medical applications. Mucins consist of different but repetitive domains and are capable of polymerizing further by forming disulfide bridges. To better understand the dynamics of mucus net forming and its structural properties it is essential to study a multi-chain system with interactions between different domains evaluated accordingly [5]. Coarse-grained off-lattice models were designed to study multi chain systems. The first one was a Rouse-like model where beads were connected by harmonic springs while the second one features fixed bond-length [6]. Two different Monte Carlo sampling algorithms based on the Metropolis scheme were applied to these models: with single bead displacements for the first model and with backrub moves for the second model. The comparison of the efficiency of both algorithms and the check of their applicability to studies of chain dynamics were the main goals of this study. The usage of these tools for studies of macromolecular films was evaluated. 
The organization of this paper is as follows. In the next section entitled Models and Simulation Methods we describe the assumptions of the model chain used. Both simulation algorithms are also presented in detail. In the next part of the paper Results and Discussion we present and discuss the simulation results concerning the chain structure and dynamics. In the last section entitled Conclusions the most important and concluding remarks are presented.

\section{MODELS AND SIMULATION METHODS}

We employed two sampling schemes that differ in the potentials that were utilized as well as the way that conformational space was sampled, i.e. chain's segments are moved. In both cases the polymer chains are represented as a sequence of $N$ connected spherical beads each of identical size and mass (homopolymer chains). All segments of the chain interact according to a repulsive excluded volume potential defined as:

$$
U_{i j}= \begin{cases}E & \text { for } \quad r_{i j} \leq d_{k} \\ 0 & \text { for } \quad r_{i j}>d_{k}\end{cases}
$$

where $E$ is an arbitrary energy barrier, $r_{i j}$ is the distance between $i^{\text {th }}$ and $j^{\text {th }}$ polymer bead and $d_{k}$ is the bead's diameter [1].

The initial conformations of chains were obtained either as a result of previous simulations or as a self-avoiding offlattice random walk that enforced excluded volume throughout the walk. Nevertheless, the starting conformation should not affect final results given the sampling algorithm is ergodic. Both methods defined a single Monte Carlo time step as a single move attempt for each bead on average.

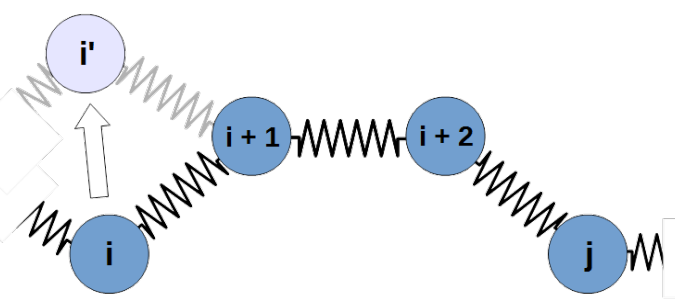

Fig. 1. In the Rouse model a single bead is displaced at random, which might cause the springs (bonds) to compress or stretch

The first of the implemented algorithms is a direct realization of the Rouse theory with the exception of the fact that excluded volume had been introduced to the system. Bonds between adjacent neighbors along the chain were enforced via harmonic energy defined as follows:

$$
U_{i j}^{S}=k\left(l_{i j}-l_{0}\right)^{2}
$$

where $l_{0}$ is the average bond length. The Monte Carlo move mimicking the Rouse model dynamics random nature was employed as a single bead displacement in a random direction as shown in Fig. 1. In this algorithm a single bead at position $\boldsymbol{r}_{i}$ was chosen at random and three random numbers $n_{x}, n_{y}$, $n_{z} \in\left[0, n_{\max }\right]$ were generated such that the new position is denoted as $\boldsymbol{r}_{i}+\left[n_{x}, n_{y}, n_{z}\right]$ and $\sqrt{n_{x}^{2}+n_{y}^{2}+n_{z}^{2}}=1$ (arbitrary unit) with $l_{0}=3.8$ to put the number into perspective.

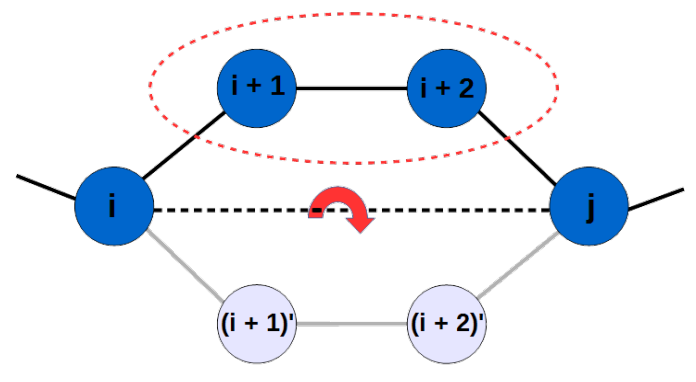

Fig. 2. In the backrub model two beads are chosen and the entire chain fragment between them is swung around a rotation axis created between said beads

The second algorithm - a generalized backrub move explicitly enforces a constant bond length so the bonding potential was omitted here [7-11]. The move consists of choosing a pair of flanking segments and rotating the chain's fragment between them about an axis defined by said segments (Fig. 2). Originally this motion was designed for atomistic protein simulations where one residue along the backbone was moved with the rotation axis defined between $\mathrm{C} \alpha$ atoms. The general approach considers moving any number of residues but was tailored in such a way that only six of protein's internal degrees of freedom were changed no matter how big the moved chunk was, namely the $\phi$ and $\psi$ angles at both pivot points and the $\mathrm{N}-\mathrm{C} \alpha-\mathrm{C}$ bond angle, $\alpha$, at both pivots. It is indeed a different sampling philosophy of creating big conformational changes despite disturbing only few integral degrees of freedom rather than moving each segment independent of others as it was in the Rouse model. In our implementation we used a mixed algorithm which utilized both local moves restricted to up to three residues displaced, and a bigger move where up to ten segment's positions were changed at once. There was no restriction on the rotation's angle $\tau$ imposed hence the angle was drawn from a uniform distribution such that $\tau \in[-\pi,+\pi]$.

To ensure the algorithm ergodicity and enable the endof-chain segments to move we introduced a tail-bead move which was realized as follows. We generated a random vector on a sphere from uniform distribution and then normalized 
it to match the bond's length. Then we recreated the tail segment adding a new vector to the tail's neighbors coordinates thus giving it complete freedom of movement independent of the chain's interior conformation.

Even in such a simple case, both algorithms performance and even behavior is obviously dependent on the choice of parameters. In the case of the backrub motion the number of segments moved as well as the maximum rotation angle presents a well-known trade-off between the extension of conformational change and moves acceptance rate which is also the case for the Rouse model and the maximum displacement per move allowed. While one could argue that because of this the results are skewed, it is still reasonable to compare the methods qualitatively within reasonable parameter's choice.

\section{RESULTS AND DISCUSSION}

In order to assert our models correctness and compare the results between both models we performed a series of simulation tests. The Rouse model which has been thoroughly investigated both theoretically and by Molecular Dynamics simulations provides us with well defined characteristics that simple polymer systems follow [1, 12]. Several scaling laws are considered valid and thus can serve as means for our tests of both static and dynamic properties of our models.

\section{1. Static properties}

Static properties of chains in infinite diluted solutions (at good solvent conditions, i.e. well above the $\theta$ temperature) are considered to obey self-avoiding random (SAW) walk statistics. Parameters often used to characterize the size of macromolecules are the mean-squared end-to-end distance and the mean-squared radius of gyration. For a bead-spring model consisting of $N$ beads of equal mass (and with mass $m=1)$ the mean-squared end-to-end distance $\left(R^{2}\right)$ and the mean-squared radius of gyration $\left(R_{g}^{2}\right)$ are defined as follows:

$$
\begin{gathered}
\left\langle R^{2}\right\rangle=\left\langle\left(r_{N}-r_{1}\right)^{2}\right\rangle \\
\left\langle R_{g}^{2}\right\rangle=\left\langle\sum_{i=1}^{N}\left(r_{i}-r_{C M}\right)^{2}\right\rangle
\end{gathered}
$$

where $\boldsymbol{r}_{C M}$ is the chain's center of mass. For SAW's both of these parameters obey scaling laws $R_{g}^{2}, R^{2} \sim N^{2 \nu}$, where the parameter $n$ for three dimensions should be $\nu \approx 0.5888$ [1214]. Checking if a model can reproduce the scaling behavior can be considered a validation test. It has been proven, however, that even in the case of bead-spring models the parameters used to characterize it, namely the bond equilibrium length $\left(l_{0}\right)$ and the effective bead diameter $\left(d_{0}\right)$ can influence the chain's behavior $[15,16]$. Based on that knowledge we tested different ratios of $l_{0} / d_{0}$ by changing the bead's size while maintaining the same bond's length to achieve proper size scaling as shown in Fig. 3. We chose the ratio to reflect the scaling law but, most importantly, to achieve the same exponent for both models as the goal was to test their dynamic properties and compare them. While both models behave somewhat differently it is possible to find the right scaling value within a reasonable $l_{0} / d_{0}$ ratio range. Given the difference in bead size it is not surprising that the Rouse model exhibits slightly higher $R_{g}^{2}$ and $R^{2}$ values while both models follow the scaling law which can be seen in Fig. 4. We achieved $R_{g}^{2}$ and $R^{2} \sim N^{1.20}$ which is in very good agreement with theory and simulations of other models [12, 17-19] but, as shown in Fig. 3, this is a matter of choice rather than a built-in property of the models, and the important conclusion is that results for both algorithms fit the linear scaling very well in a range of chain lengths.

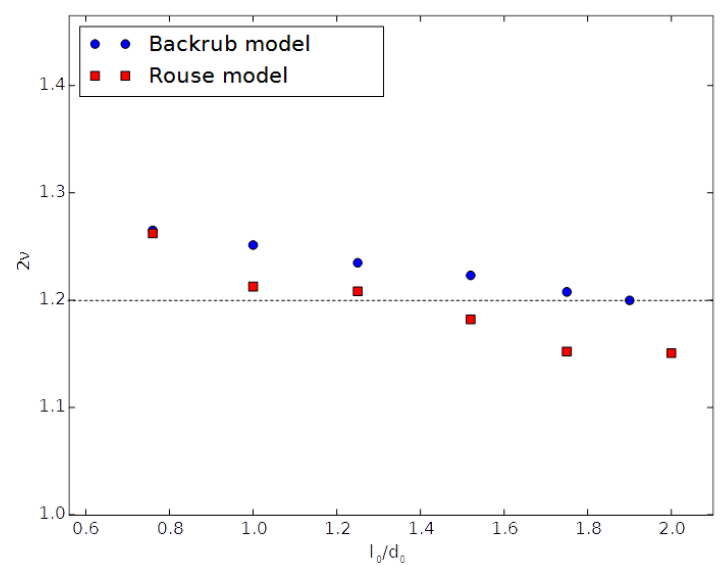

Fig. 3 . The scaling exponent $2 n$ obtained for the bead-spring model as a function of the $l_{0} / d_{0}$ ratio

\section{2. Dynamic properties}

Within the framework of the Rouse model diffusion can be described as a Brownian motion process where except for the bonded interactions particles move independently of each other through collision with the solvent particles where the thermal force driving the process is implied. The process is random in nature and thus can be modeled as a random walk. The speed at which a single particle diffuses is described in general by the mean square displacement (MSD) function defined as:

$$
\mathrm{MSD}=\left\langle(r(t)-r(0))^{2}\right\rangle,
$$

where $\boldsymbol{r}(t)$ denotes the particle's position vector in given time $t[12,17]$. The behavior of this function is well defined within the Rouse model of macromolecules and two particular cases are of interest: the center of mass and middle segment displacements [20, 21]. It is known that for a polymer chain the displacement is increasing with time linearly MSD $\sim t$ and 


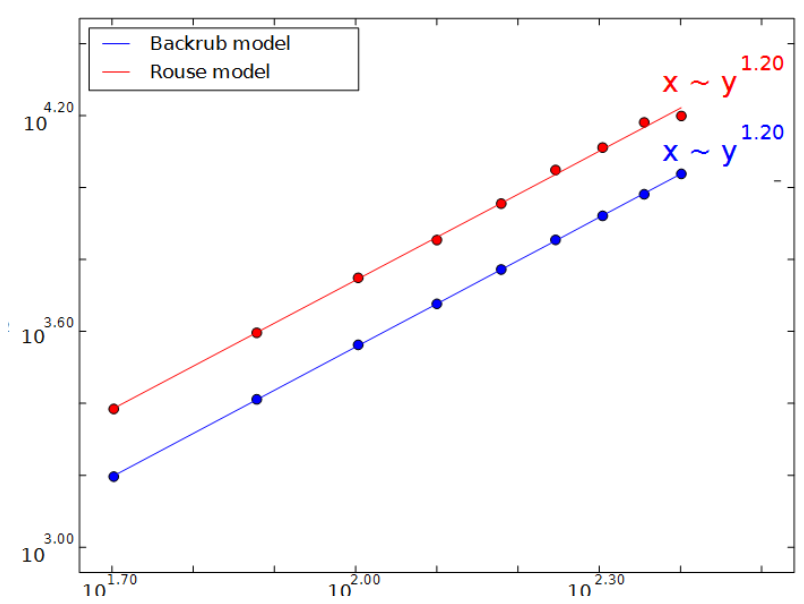

(a)

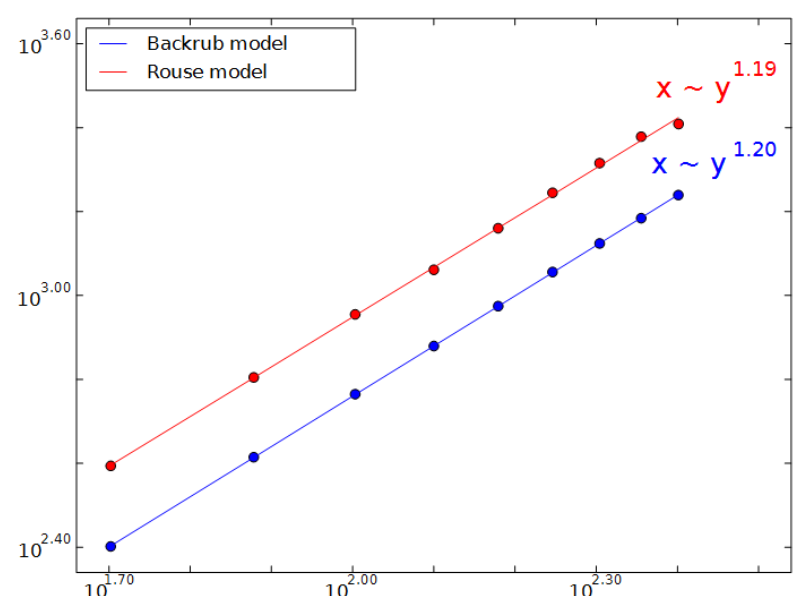

(b)

Fig. 4. The mean-squared end-to-end distance $<R^{2}>$ (a) and the mean-squared radius of gyration $<R_{g}^{2}>$ (b) as a function of the chain's length $N$ in a log-log scale

it is also how the chain's center of mass behaves [12, 17]. This is not the case, however, for the middle segment which follows two time regimes. Defining the middle segment displacement function as $g_{1}(t)$ and the longest relaxation time as $\tau_{R}$ :

$$
\begin{gathered}
g_{1}(t) \sim t^{1 / 2} \quad \text { for } \quad t<\tau_{R}, \\
g_{1}(t) \sim t^{1} \quad \text { for } \quad t \geq \tau_{R} .
\end{gathered}
$$

MSD behavior of a single chain for both models is shown in Fig. 5. One can see the expected linear time dependency $g_{C M}(t) \sim t^{1.00}$ for the center of mass diffusion and two time regimes for the middle segment displacements with $g_{1}(t) \sim$ $t^{0.60}$ initially which gradually shifts to $g_{1}(t) \sim t^{1.00}[12]$. The preliminary value is slightly higher than the theoretical

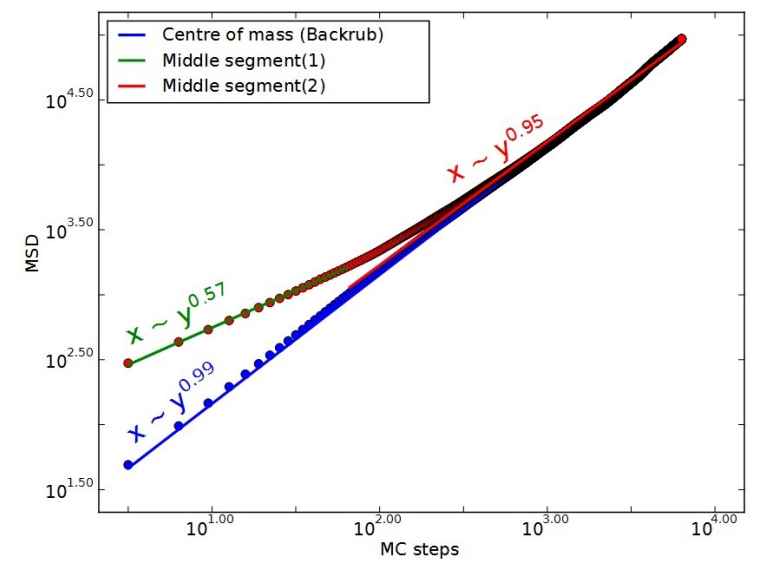

(a) prediction but the crossover can be observed and is commonly interpreted as a transition between the initial chain internal rearrangements and a free diffusion along the chain's contour which takes place after the longest relaxation time $\left(\tau_{R}\right)$ is achieved. It is worth noting that in our implementation of the Rouse model disabling the excluded volume potential leads to achieving middle segment displacement data that fits the theory better.

The other relevant parameter is the diffusion coefficient $D$ which describes the speed at which the diffusion happens. One can calculate the coefficient using the equation:

$$
D=\frac{\left\langle(r(t)-r(0))^{2}\right\rangle}{6 t}
$$

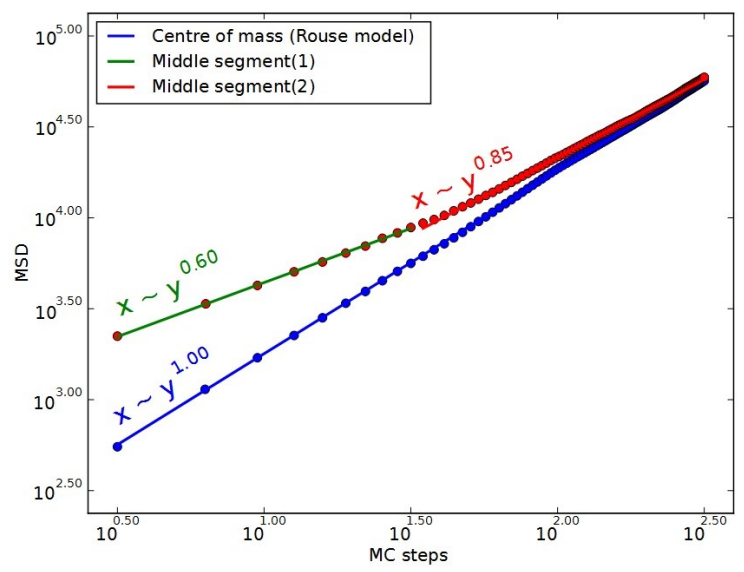

(b)

Fig. 5. Log-log plot of the autocorrelation functions of the center-of-mass and $g_{1}(t)$ for Backrub (a) and Rouse (b) algorithms 


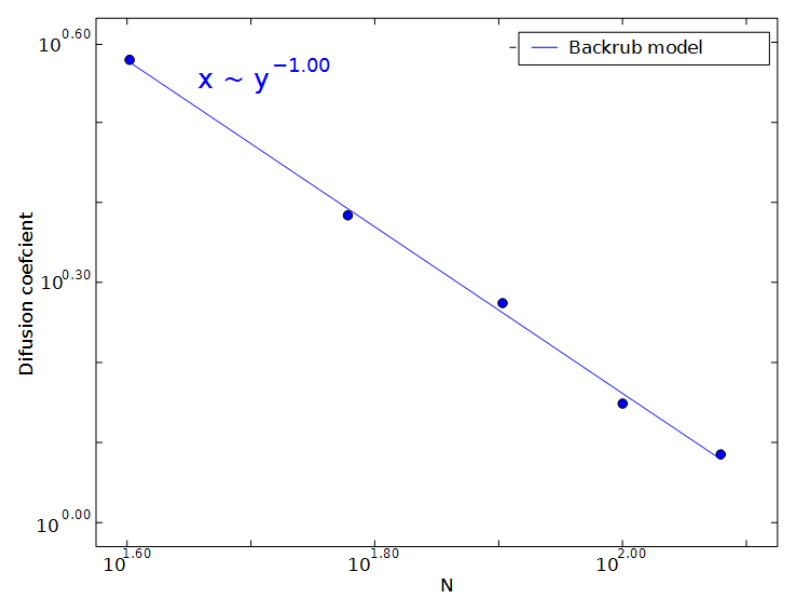

(a)

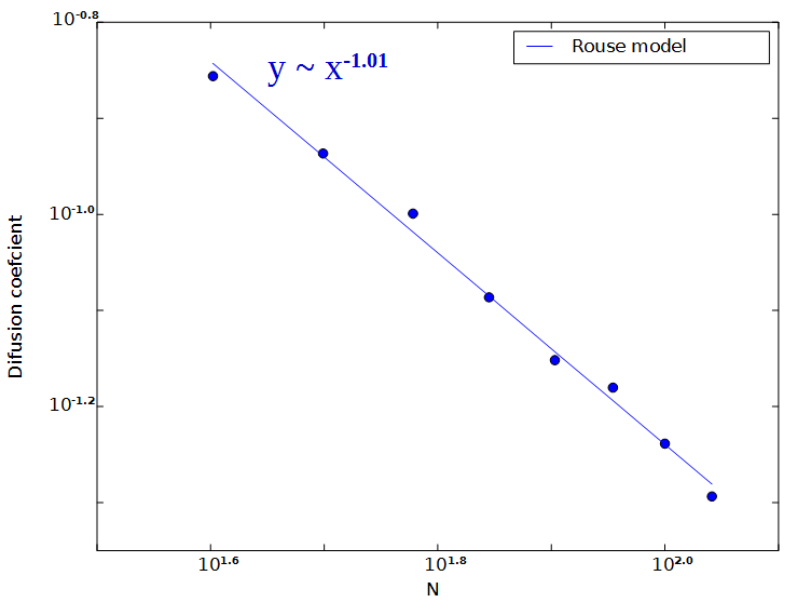

(b)

Fig. 6. Log-log plot of diffusion coefficient $D$ as a function of the chain's length $N$ for both Backrub (a) and Rouse (b) algorithms

The Rouse model is solvable and given that the dynamic viscosity $\eta$ is proportional to the number of beads $N$ gives the exact result for $D$ depending on temperature and friction coefficient $\zeta$ [22]:

$$
D=\frac{k_{B} T}{N \zeta}
$$

The above equation makes it clear that the diffusion coefficient also scales with the number of beads and within the Rouse model framework the dependency is given by another power law $D \sim N^{-1}$ [12]. Both our models were able to replicate this result, as shown in Fig. 6, with data points fitting the linear curve rather well for the chosen range of chain lengths which indicates that the pseudo-time scale has been properly chosen for both models.

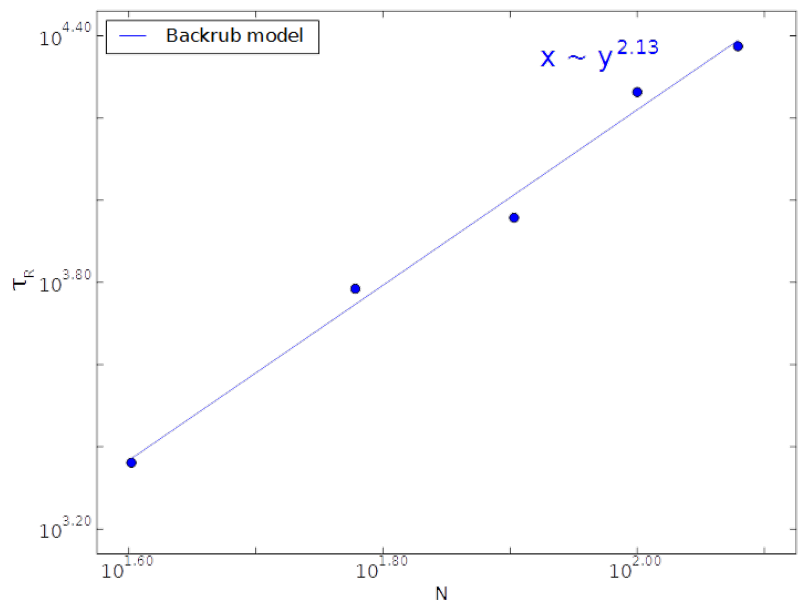

(a)
We have also examined a third dynamic property of polymer chains which is the longest relaxation time and its scaling with the chain's length. One can define an autocorrelation function $g_{R}(t)$ based on the mean-squared end-to-end distance:

$$
g_{R(t)}=\frac{\langle R(0)\rangle^{\circ}\langle R(t)\rangle}{\left\langle R^{2}\right\rangle}
$$

The longest relaxation time $\tau_{R}$ itself is then calculated using the autocorrelation function:

$$
g_{R}(t) \exp \left(\frac{-t}{\tau_{R}}\right)
$$

Scaling of the longest relaxation time is again a well-known parameter with $\tau_{R} \sim N^{2.2}$ for systems considered in this

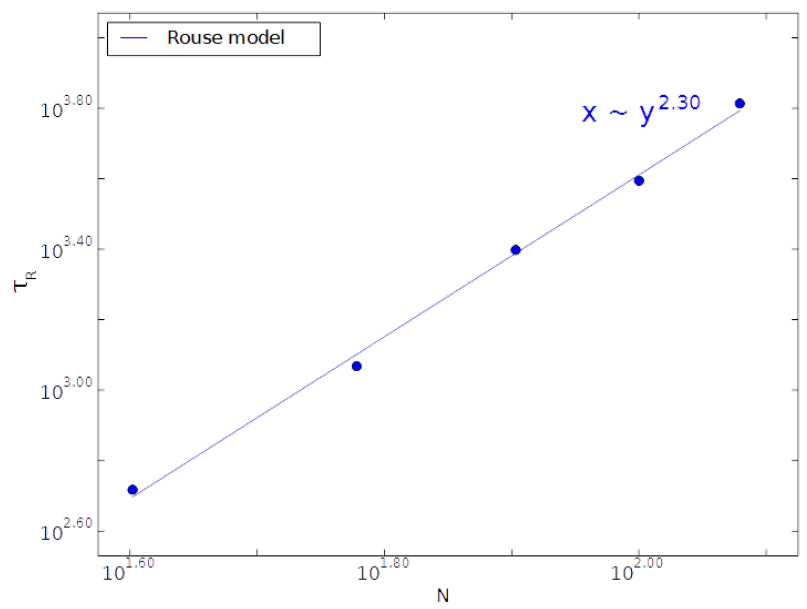

(b)

Fig. 7. Log-log plot of the mean-squared end-to-end distance relaxation time as a function of chain length for Backrub (a) and Rouse (b) algorithms 
work [12]. Our results for both models can be seen in Fig. 7 with scaling slightly differing from the theoretical exponent. Interestingly, the divergence is different in both cases with the backrub model having a slightly weaker than expected time-relaxation dependence and the Rouse model a stronger one, both within a reasonable margin from the commonly accepted values, however. Therefore, the backrub algorithm seems to be a tool than is able to reproduce the dynamic behavior of polymer chains.

Comparing computational efficiency of two algorithms in a complex environment such as a Monte Carlo polymer simulation is a task in itself and we feel that it goes beyond the scope of this paper. Not mentioning the fact that there is no simple unit of performance measure one has to consider questions such as: how well will the algorithm do in different polymer concentrations? How much performance can one gain by implementing common tools such as a linked list for neighbor detection? Considering these and other concerns one has to choose an algorithm that fares better in the problem they are trying to solve. In the case of a single chain one can easily see that the naive Rouse model implementation is more efficient with the exact numbers depending on the algorithms parameter choice considering reasonable parameters discussed in the previous section.

\section{CONCLUSIONS}

We performed Monte Carlo simulations of two different off-lattice models of a polymer chain using two different simulation algorithms which seemed to be appropriate for a given model. Therefore, the bead-spring model was simulated by a classical sampling algorithm (one-bead random displacements) while the model with constant bond length was simulated using the backrub algorithm (rotation of a rigid chain's fragment). The main goal of these simulations was the evaluation of the backrub model usefulness and efficiency. The possibility of studying the dynamic properties of macromolecules by means of this algorithm was also checked.

We have evaluated both static and dynamic properties of the models and compared them with previous simulations and theoretical predictions. In case of static properties the obtained size to chain length scaling for both sampling schemes was correct with quantities such as the mean-squared radius of gyration and the mean-squared end-to-end distance studied. Dynamic properties of our models were tested by evaluating mean square displacements and self-diffusion coefficients. While the short time MSD values diverged slightly from the theoretical predictions, we achieved proper qualitative behavior as well as correct diffusion coefficients with both models exhibiting very similar characteristics.

We may, therefore, conclude that both presented models are well fit for Monte Carlo simulations of dilute homopolymer systems but further investigation is necessary to determine their usefulness in more complex environments and potential underlying issues with short time diffusion behavior.

\section{Acknowledgment}

The computational part of this work was done using the computer cluster at the Computing Center of the Department of Chemistry, University of Warsaw. This work was supported by the Polish National Science Center grant UMO2013/09/B/ST5/00093.

\section{References}

[1] J. Baschnagel, J.P. Wittmer, H. Meyer, Monte Carlo Simulation of Polymers: Coarse-Grained Models, in: Computational Soft Matter: From Synthetic Polymers to Proteins, Lecture Notes, N. Attig, K. Binder, H. Grubmüller, K. Kremer (eds.), John von Neumann Institute for Computing, Jülich, Vol. 23, p. 83-140, 2004.

[2] G.J. Strous, J. Dekker, Mucin-type glycoproteins, Crit. Rev. Biochem. Mol. Biol. 27, 57-92 (1992).

[3] D.J. Thornton, J.K. Sheehanm, From mucins to mucus: toward a more coherent understanding of this essential barrier, Proc Am. Thorac. Soc. 1, 54-61 (2004).

[4] M. A.Hollingsworth, B.J. Swanson, Mucins in cancer: protection and control of the cell surface, Nature Rev. Cancer $\mathbf{4}$, 45-60 (2004).

[5] P. Gniewek, A. Kolinski, Coarse-Grained Monte Carlo Simulations of Mucus: Structure, Dynamics, and Thermodynamics, Biophys. J. 99, 3507-3516 (2010).

[6] K. Binder, A. Milchev, Off-lattice Monte Carlo methods for coarse-grained models of polymeric materials and selected applications, J. Comput.-Aided Mater. 9, 33-74 (2002).

[7] M.R. Betancourt, Efficient Monte Carlo trial moves for polypeptide simulations, J. Chem. Phys. 123, 174905 (2005).

[8] M.R. Betancourt, Optimization of Monte Carlo trial moves for protein simulations, J. Chem. Phys. 134, 014104 (2011).

[9] C.A. Smith, T. Kortemme, Backrub-like backbone simulation recapitulates natural protein conformational variability and improves mutant side-chain prediction, J. Mol. Biol. 380, 742-756 (2008).

[10] A.A. Podtelezhnikov, D.L. Wild, Exhaustive Metropolis Monte Carlo sampling and analysis of polyalanine conformations adopted under the influence of hydrogen bonds, Proteins: Struct., Funct., Bioinf. 61, 94-104 (2005).

[11] I.W. Davis, W.B. Arendall III, D.C. Richardson, J.S. Richardson, The backrub motion: how protein backbone shrugs when a sidechain dances, Structure 14, 265-274 (2006).

[12] I. Teraoka, Polymer Solutions. An Introduction to Physical Properties, Wiley-Interscience, New York 2002.

[13] B. Li, N. Madras, A.D. Sokal, Critical exponents, hyperscaling, and universal amplitude ratios for two- and threedimensional self-avoiding walks, J. Stat. Phys. 80, 661-754 (1995).

[14] K. Binder, Applications of Monte Carlo methods to statistical physics, Rep. Prog. Phys. 60, 487-559 (1997).

[15] C.F. Abrams, K. Kremer, The effect of bond length on the structure of dense bead-spring polymer melts, J. Chem. Phys. 115, 2776-2785 (2001).

[16] C.F. Abrams, K. Kremer, Effects of excluded volume and bond length on the dynamics of dense bead-spring polymer melts, J. Chem. Phys. 116, 3162-3165 (2002). 
[17] D.P. Landau, K. Binder, A Guide to Monte Carlo Simulations in Statistical Physics, $3^{\text {rd }}$ edition, Cambridge University Press, Cambridge, chapter 6.6, 2009.

[18] P. Romiszowski, A. Sikorski, Dynamics of polymer chains in confined space. A computer simulation study, Physica A 357, 356-363 (2005).

[19] P. Polanowski, J.K. Jeszka, A. Sikorski, Dynamic properties of linear and cyclic chains in two dimensions. Computer simulation atudies, Macromolecules 47, 4830-4839 (2014).
[20] L. Harnau, R.G. Winkler, P. Reineker, On the dynamics of polymer melts: Contribution of Rouse and bending modes, Europhys. Lett. 45, 488-494 (1999).

[21] D. Panja, Generalized Langevin equation formulation for anomalous polymer dynamics, J. Stat. Mech. Theor. Exp. L02001 (2010).

[22] K. Kremer, G.S. Grest, Dynamics of entangled linear polymer melts: A molecular-dynamics simulation, J. Chem. Phys. 92, 5057-5086 (1990).
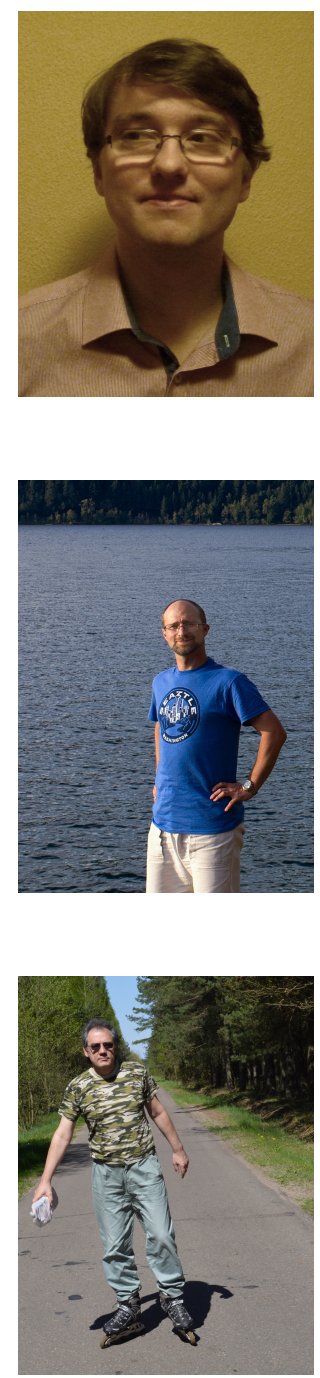

Andrzej Sikorski graduated from the Department of Chemistry, University of Warsaw in 1980. He completed his PhD Thesis in Chemistry in 1986 in the same Department. In 1987/89 and 1990/91 he worked as a postdoc and visiting professor in Washington University in St. Louis and in The Scripps Research Institute in La Jolla. In 1995 he obtained DSc degree in Chemistry. His main scientific interest is the theory and simulation of polymers at interfaces and in confined geometries.
Aleksander Kuriata received his MSc degree in Chemistry from the University of Warsaw in 2015. Currently he is a PhD candidate at the Laboratory of Theory of Biopolymers at the University of Warsaw. His research interests include polymer and biopolymer theory and simulations.

Dominik Gront received his $\mathrm{PhD}$ in 2006 from the Faculty of Chemistry, University of Warsaw. After postdoctoral training at the University of Virginia, Charlottesville, VA and University of Washington, Seattle, WA, he joined back the Faculty of Chemistry, University of Warsaw. In his research he focuses on novel coarse-grained methods for biomacromolecular modeling. He actively develops Rosetta and BioShell software packages. 